\title{
Time-resolved surface magnetometry in the nanosecond scale using synchrotron radiation
}

Cite as: Journal of Applied Physics 83, 1563 (1998); https://doi.org/10.1063/1.366866

Submitted: 15 July 1997 . Accepted: 25 October 1997 . Published Online: 04 June 1998

F. Sirotti, R. Bosshard, P. Prieto, G. Panaccione, L. Floreano, A. Jucha, J. D. Bellier, and G. Rossi

View Online

\section{ARTICLES YOU MAY BE INTERESTED IN}

Observation of surface enhanced multiphoton photoemission from metal surfaces in the short pulse limit

The Journal of Chemical Physics 102, 8606 (1995); https://doi.org/10.1063/1.468962

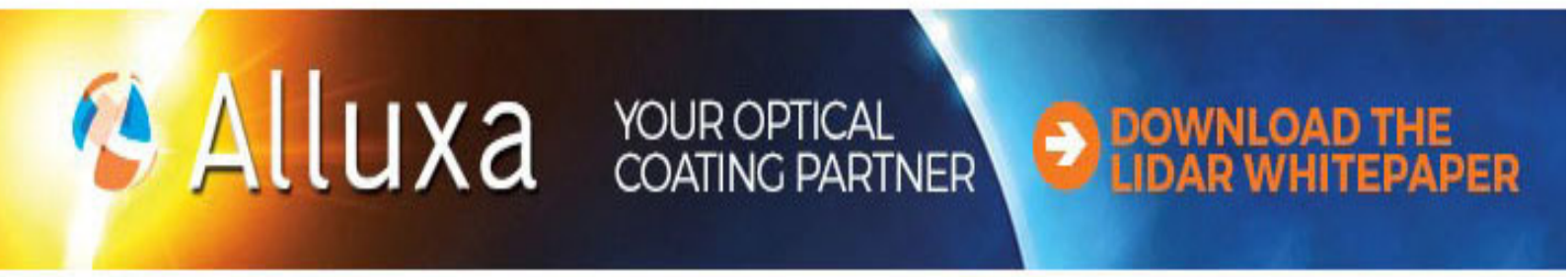




\title{
Time-resolved surface magnetometry in the nanosecond scale using synchrotron radiation
}

\author{
F. Sirotti, ${ }^{\text {a) }}$ R. Bosshard, P. Prieto, ${ }^{\text {b) }}$ and G. Panaccione ${ }^{\mathrm{c})}$ \\ Laboratoire pour l'Utilisation du Rayonnement Electromagnetique, CNRS-CEA-MESR, \\ F-91405 Orsay, France \\ L. Floreano \\ INFM-TASC Laboratory, Area di Ricerca 99 Padriciano, I-34012 Trieste, Italy
}

A. Jucha and J. D. Bellier

Laboratoire pour l'Utilisation du Rayonnement Electromagnetique, CNRS-CEA-MESR, F-91405 Orsay, France

G. Rossi

Laboratorium für Festkörperphysik, ETH-Zürich, CH-8093, Switzerland; INFM-Dipartimento di Fisica, Universitá di Modena, I-41100 Modena, Italy

(Received 15 July 1997; accepted for publication 25 October 1997)

The dynamics of magnetization reversal at surfaces and interfaces is studied by a specific photoelectric effect experiment with pulsed soft $\mathrm{x}$ rays. This novel time-resolved surface magnetometry is based on the measure of the spin polarization of the total photoejected electron yield by means of a Mott scattering experiment and on the acquisition synchronism with the multibunch structure of the positron storage ring SuperAco at Orsay (500 ps pulses at $120 \mathrm{~ns}$ intervals). We present results on the fast magnetization reversal of Fe ultrathin films deposited on amorphous low-coercivity ferromagnetic ribbons. (c) 1998 American Institute of Physics.

[S0021-8979(98)07303-4]

\section{INTRODUCTION}

Understanding the dynamics of magnetization at surfaces and interfaces is a prerequisite for a better description of fundamental and domain-mediated magnetic phenomena, ${ }^{1-3}$ as well as for the tailoring of specific magnetic devices with the aim to increase the magnetic data exchange rate.

The effective coercivity of a ferromagnet depends upon the time scale of the process used to induce the magnetization reversal: by standard methods like vibrating -sample and $60 \mathrm{~Hz}$ magnetometers the time scale of $1 \mathrm{~s}$ and $10^{-5}$ $-10^{-6} \mathrm{~s}$ respectively are accessible. ${ }^{1,4}$ Experiments with $10^{-6} \mathrm{~s}$ resolution have been done on thin magnetic films by magnetooptical measures. ${ }^{3,5}$ The technologically relevant time scale of $10^{-8} \mathrm{~s}$ has been reached by electrical measurements. ${ }^{6,7}$ All of these techniques are sensitive to bulk magnetic properties and cannot be applied to surfaces, interfaces and atomically thin films (ATF). Actually the current trends in magnetism imply the study and the exploitation of mesoscopic particle size and atomically thin films in artificial structures in order to improve the recording resolution, the signal to noise ratio and the magnetic stability as well as the practical use of the specific magnetic properties of lowdimensional systems and of the stacking of atomically thin layers. A surface science approach to magnetometry is therefore quite appropriate, particularly if the surface sensitivity,

\footnotetext{
a)Electronic mail: sirotti@LURE.U-PSUD.FR

${ }^{b)}$ Present address: Dept. Fisica Aplicada, C-XII, Fac. De Ciencias, Univ. Autonoma de Madrid, 28049 Cantoblanco, Spain.

${ }^{c}$ Present address: Institut de Physique, Univ. Neuchâtel, CH-2001 Neuchâtel Switzerland.
}

and the sensitivity to small amounts of ferromagnetic matter can be coupled with time resolution on the relevant time scale.

We present in this article the principles and the demonstration of a novel approach to time resolved surface magnetometry based on spin polarization (SP) measurements of the time-resolved photoemission with two time scales accessible as a consequence of the photoexcitation source properties: single pulse resolution of $500 \mathrm{ps}$ and real time measurements at $120 \mathrm{~ns}$ time intervals.

The photoexcitation process occurs on the $10^{-18} \mathrm{~s}$ time scale and the ejection of the so-called secondary electrons (i.e., Auger and scattered electrons) occurs within $10^{-15} \mathrm{~s}$ from the primary photoexcitation process so that the actual time resolution of a method based on the photoelectric effect is entirely set by the time duration of the excitation light pulse. The shortest available light pulses are from laser sources, routinely operated at ps or even sub-ps times. Pioneering experiments based on SP measurements with ps time resolution from a dye laser investigated the spin-lattice relaxation in $\mathrm{Gd}$ and $\mathrm{Fe}$ films. ${ }^{8,9}$ The main limit to the application of the laser pulses comes from the photon energy, which is usually too low to photoexcite above the vacuum level of the material thus requiring special techniques for reducing the sample work function. Moreover, the maximum repetition rate is also limited. Synchrotron radiation (SR) from storage rings provides pulses of some tens of ps duration (30-50 ps from third generation sources, $200-500 \mathrm{ps}$ from second generation sources like the one used in the present experiments) with an energy spectrum extending to $\mathrm{x}$-ray energies and with repetition rates set by the operation 
mode of the storage ring, i.e., from a few ns up to several hundreds of ns. The surface sensitivity of this photoemission probe is achieved by the adequate energy selection of the electrons to be detected. The sensitivity to magnetism is then reached by a Mott scattering experiment which measures the SP of the ejected electron signal, without affecting the time scale of the experiment. ${ }^{10}$ The magnetometry based on the SP measurement of secondary electrons photoexcited by $\mathrm{SR}^{11}$ can fit the time domain relevant for many collective surface magnetic phenomena like reversal and reorientation processes, structural phase transitions, interdiffusion processes, growth processes, surface melting, etc. We report on the results obtained on the fast magnetization reversal of atomically thin Fe layers deposited in ultrahigh vacuum onto low-coercivity ferromagnetic ribbons.

\section{EXPERIMENT}

The time basis of the measurements was provided by the two-bunch operation of the SuperAco positron storage ring at Orsay providing pulses of 500 ps full width, at $120 \mathrm{~ns}$ intervals. The radiation source was the DOMINO undulator operated in wiggler mode illuminating the SU 7 beam line equipped with a TGM monochromator that was operated in total reflection mode therefore yielding polychromatic pulses of soft $x$ rays. The photoelectrons ejected from the surface of the ferromagnetic sample after each SR pulse were accelerated to $100 \mathrm{kV}$ kinetic energy and subsequently spin analyzed, along the direction specified by the magnetizing field, by means of a Mott scattering experiment ${ }^{12}$ performed with a $100 \mathrm{kV}$ Mott detector of the ETH-Zürich type. ${ }^{13}$ Details of a Mott scattering setup can be found elsewhere. ${ }^{14}$ The basic features of SP magnetometry with a Mott detector imply: (a) the collection of the total yield of the ejected electrons from a metal surface as a consequence of the photohole decay, (b) the formation of an electron beam and its acceleration up to $100 \mathrm{kV}$ by means of a focusing electrostatic lens accelerator, (c) the scattering of the electron beam onto a thin gold target. Spin orbit scattering in the core electron field of the target determines a right-left asymmetry with respect to the quantization axis in space defined by the scattering plane of the Mott detector (which for our experiment coincides with the sample magnetization axis). The right-left asymmetry is defined as $A_{\text {Spin }}=\left(I_{\text {left }}-I_{\text {right }}\right) /\left(I_{\text {left }}+I_{\text {right }}\right)$ where $I_{\text {left (right) are }}$ the intensities measured by detectors positioned at $120^{\circ}$ in the plane perpendicular to the quantization axis. The measured asymmetry, after correction by the Sherman function describing the scattering process and by the instrumental asymmetry, is proportional to the SP of the electrons emitted from the sample, i.e., to its average magnetization. ${ }^{10}$ The surface sensitivity comes from the kinetic energy of the ejected electrons from the sample, which is of a few electron volts, as defined by the transmission function of the electrostatic lens accelerator.

The experiment is performed in ultrahigh vacuum (base pressure $4 \times 10^{-10}$ mbar) on samples grown in situ, at temperatures in the range $T=150-300 \mathrm{~K}$. A typical hysteresis loop obtained from SP measurements in the usual operating mode, shows a remanent SP of $\sim 15-25 \%$ (depending on Fe film thickness) and a coercive field of about $0.1 \mathrm{Oe}$, for $\mathrm{Fe}$ films of 1-5 nm thickness deposited on atomically clean (ion sputtered) surfaces of amorphous low-coercivity ferromagnetic Si-based alloys (commercial name: Vitrovac). A beam stopper, synchronized with the SP acquisition apparatus, let the surface to be exposed to the photon beam for less than one second during which time the acquisition is performed. This prevents a quick deterioration of the sample surface exposed to the high radiation power of the polychromatic SR beam. Details of the geometry and sample mounting can be found elsewhere. ${ }^{15}$

A schematic diagram of the experimental setup is shown in Fig. 1. SR impinges onto the sample at $45^{\circ}$ with respect to the surface normal. The ejected yield within an emission cone of $10^{\circ}$ around the sample normal is collected and accelerated towards the Au foil of the Mott scatterer. The clock of the experiment is referenced to the storage ring pulse structure by measuring with a pick-up induction device the passage of the particle bunches in the SuperAco storage ring: this pick-up signal is electronically shaped and transferred to the data acquisition system via an optical link. The whole measurement and data storage system is directly connected to the solid state detectors of the Mott scatterer at $100 \mathrm{kV}$ with respect to ground. The silicon surface barrier detectors (PIPS-Canberra) output a pulse amplitude proportional to the number of detected electrons. The signal is preamplified with a total pulse width of about $60 \mathrm{~ns}$ and analog to digital converted by an 8 bits flash ADC, the resulting information is written automatically at the SuperAco frequency in a First In First Out (FIFO) 8192 bytes memory. A delay stage is introduced before the optical link of the clock (pick-up) signal in order to set the correct phase relationship between the electron pulses and the trigger signal.

The setup to reverse the magnetic field at high rate is schematically represented on the left of Fig. 1. The trigger to the magnetization reversal pulse is given synchronously with the SuperAco clock signal. At the beginning of a timeresolved measurement the sample is kept in one magnetization state by flowing a steady current $\left(I_{-}\right)$through the windings. Short magnetic field pulses are obtained by closing a fast high voltage transistor switch (Belcke type). When the switch is closed an extra current, delivered by the high voltage supply HV, is flowing through the windings. The magnetization reversal is obtained by selecting the amplitude of these two opposite currents. The rising time of such kind of pulses is of the order of a few ns for a pure resistor charge. The inductance $L$, introduced by the magnetic circuit which actually creates the magnetizing field applied to the sample, increases the pulse rising time. The time evolution of the applied magnetic field is shown in Fig. 2 for few values of the pulse current. The applied magnetic field reaches a stationary condition after $70 \mathrm{~ns}$. This fact selects two classes of experiments: those in which one observes the magnetization reversal in constant applied field conditions (i.e., those for which the 100 ns raise time of $H$ is a short time with respect to the dynamical response) and those experiments which measure the response of the surface magnetization to the time-varying magnetizing field, e.g., when the reversal occurs in a few tens of ns. This last class of experiment is in fact sensitive to the time derivative of the applied field 


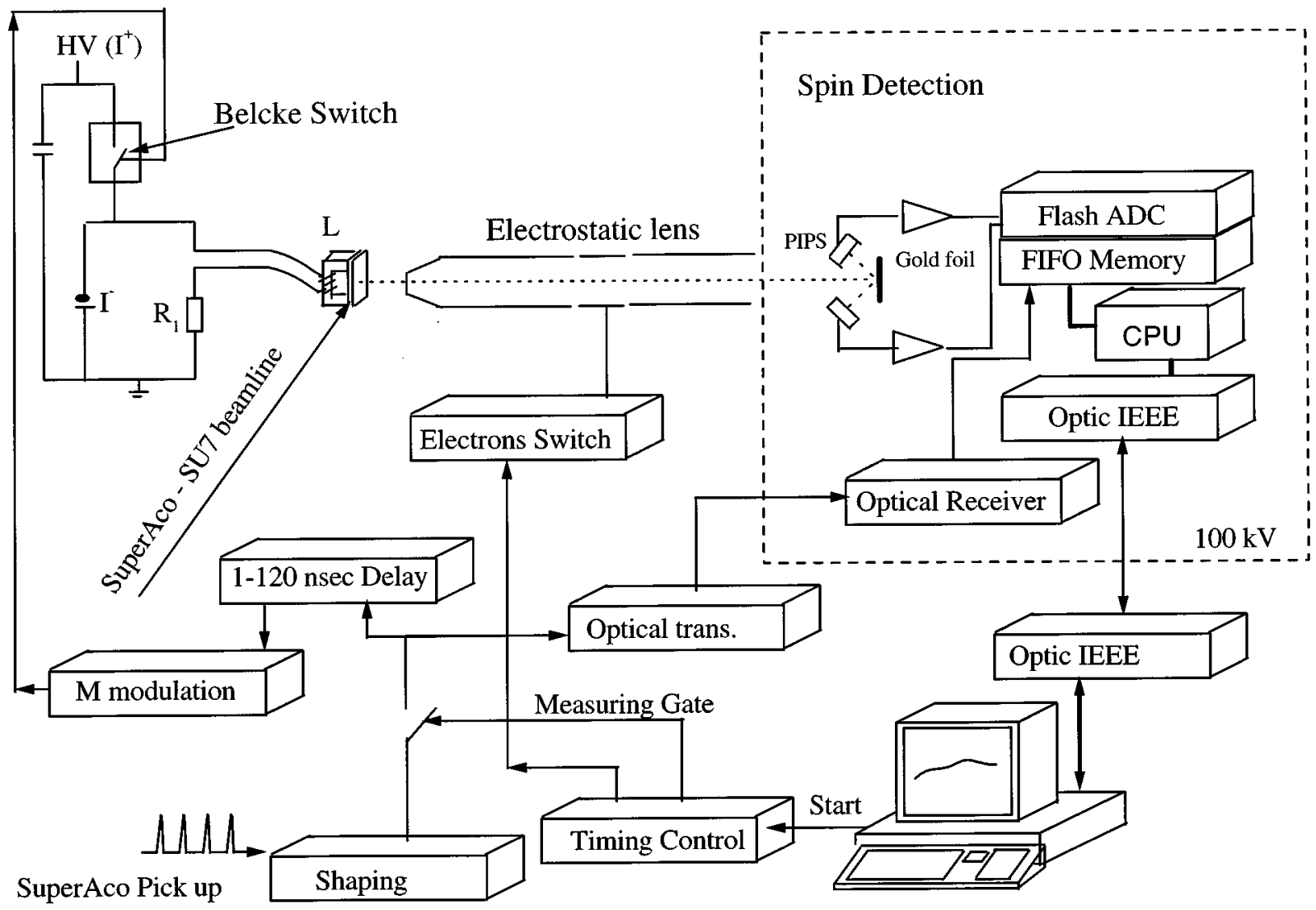

FIG. 1. Scheme of the experimental setup. Upper left: the electrical circuit for creating the magnetizing field, sample mounting, and geometry of the experiment. Right hand inset: detection electronic of the Mott detector (floating at $100 \mathrm{kV}$ ). Lower left: synchronization signal from the storage ring and timing control scheme.

$d H / d t$. The values of $d H / d t$ obtained from our magnetizing circuit are indicated in Fig. 2 (linear approximation of the raising edge). For both kind of experiments, a measuring event corresponds to the collection and the measurement of one electrons bunch created by one light pulse.

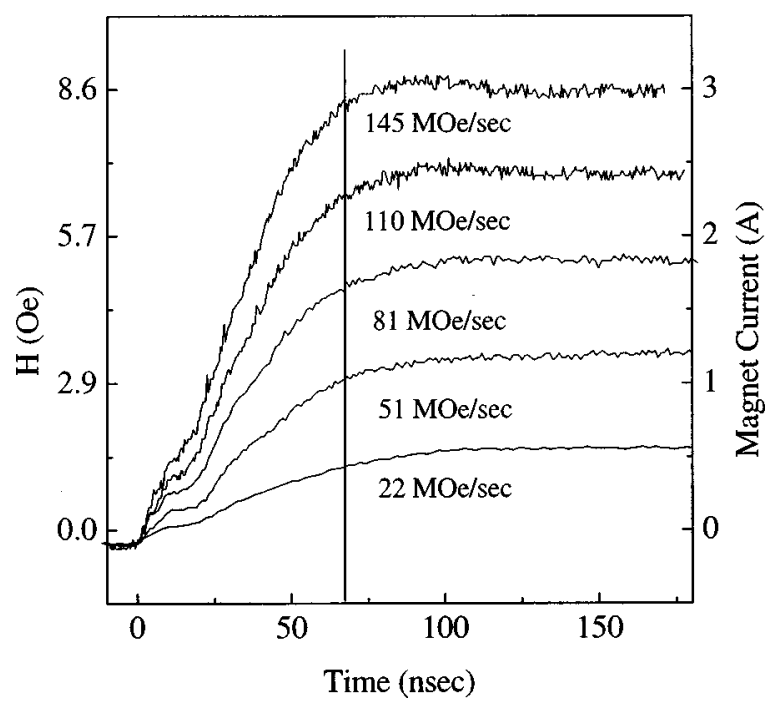

FIG. 2. Time structure of magnetizing field for different values. The applied magnetizing field reaches a constant value after $70 \mathrm{~ns}$. In the linear transient regime $(20 \mathrm{~ns}<t<60 \mathrm{~ns})$ the field gradient values are indicated.
After the trigger signal, the acquisition lasts until the FIFO memory is full, i.e., after $983 \mu$ s. Whenever the apparatus is not triggered, the electron beam is deviated from the Mott detector by an electrostatic lens; this prevents the saturation effects of the solid state detectors and electronics. The time structure of such a measuring procedure is reported in Fig. 3, as given from the timing control electronics of Fig. 1. When the computer gives the start signal, the electrons switch is opened [Fig. 3(a)] and after a delay of about $2 \mathrm{~ms}$ (to establish stationary electrostatic conditions) the measuring gate [Fig. 3(b)] enables the transmission of the SuperAco clock signal [Fig. 3(c)]. The rise of the first clock transition triggers the current pulse in the windings producing the magnetizing field. This allows to start the sequence of measuring events with a well-defined delay with respect to the current pulse (with an incertitude smaller than one ns), i.e., to perform pump-probe type experiments. The ultimate time resolution is defined by the SR pulse duration (500 ps). The shape of the magnetic pulse, measured from the resistance $R$, is shown in Fig. 3(d) in the case of a magnetic field reversal produced by switching the current in the magnetic circuit from $-300 \mathrm{~mA}$ to $3 \mathrm{~A}$. Finally, the electrons switch is closed within $1 \mathrm{~ms}$ from the end of the acquisition and the trigger transmission is disabled. The FIFO memory is restored by a 68070 microprocessor and the system is ready for a new measuring process. 

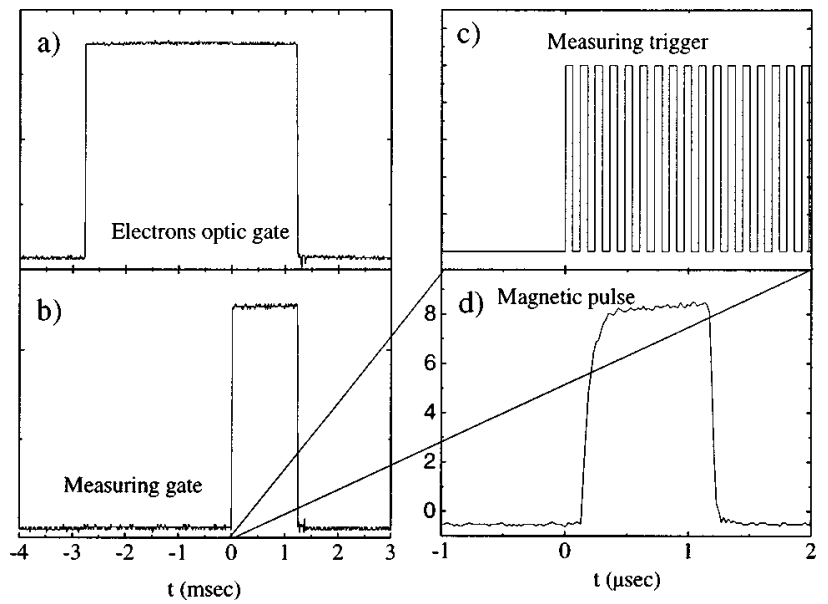

FIG. 3. Timing control of the experiment: (a) Opening of the electrons switch allowing the photoelectrons to be accelerated to the Mott detector; (b) after $2 \mathrm{~ms}$ the measuring gate enables the transmission of the trigger signal. (c) Trigger signal. (d) The magnetizing pulse $(-300 \mathrm{~mA}$ to $3 \mathrm{~A}$ in this example).

\section{RESULTS}

The presence of an external magnetic field applied to the sample during the measurements affects the trajectories of the photoejected electrons; this may result in an additional, undesired, asymmetry. Test experiments demonstrated that the SP value obtained from a magnetically saturated surface is stable even in presence of magnetic pulses exceeding the saturation value. Figure 4 shows such a test for pulses of +3 A (saturation value $<+50 \mathrm{~mA}$ ). The effect of the applied field on the scattered intensities in the left and right channels

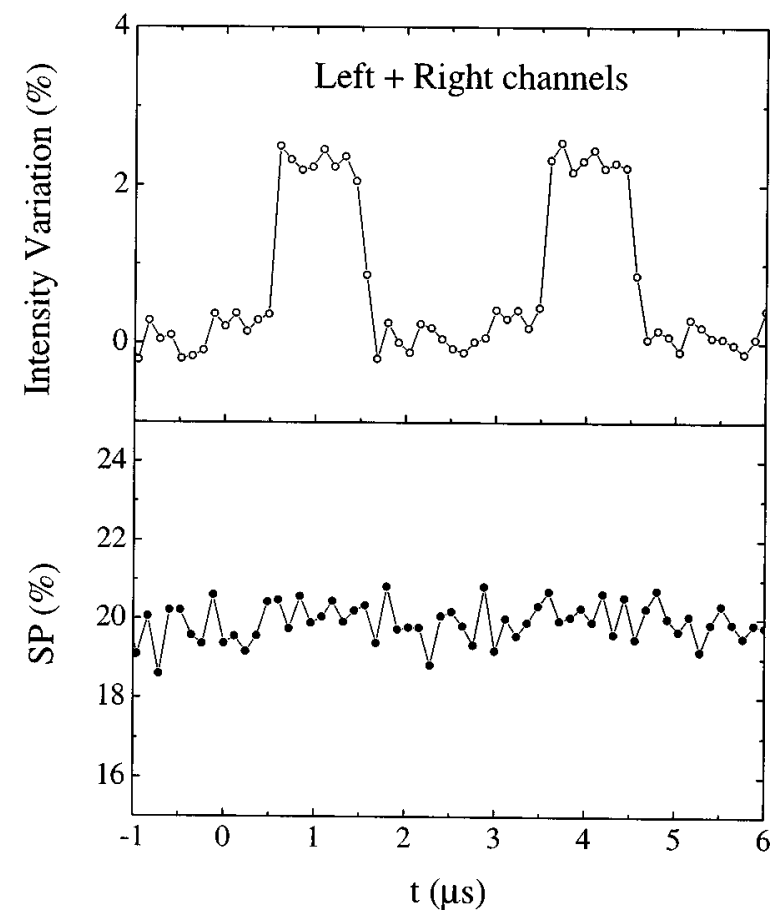

FIG. 4. Test of SP measurement with large applied fields. Top panel: variation of total count rate on detectors during $H$ field pulses of $\sim 9$ Oe. Bottom panel: the SP measure is unaffected.

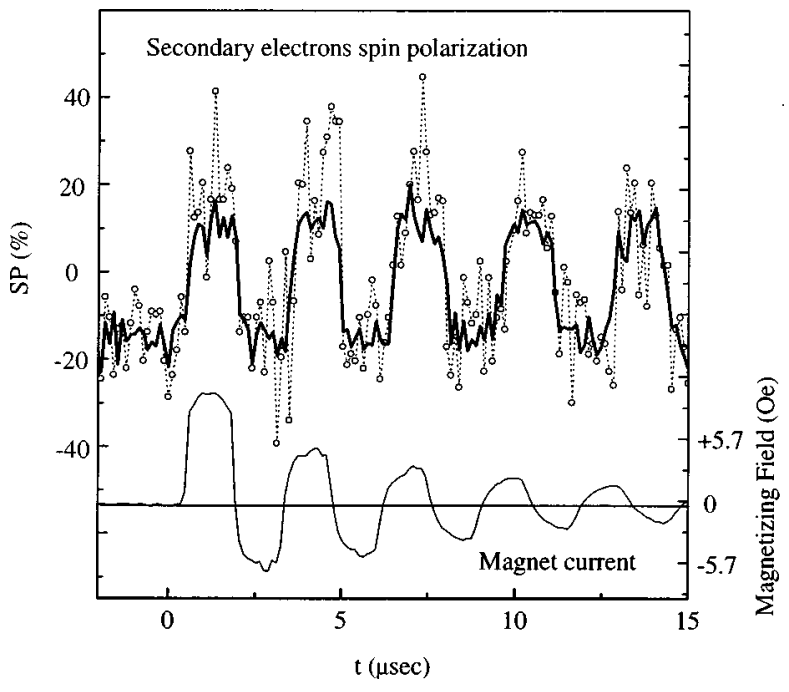

FIG. 5. Time-resolved SP measurements showing the variations of magnetization induced from the external magnetic field oscillations shown in the bottom of the figure. The values of the magnet current are reported on the $y$ scale on the right. The sample was a $15 \AA$ Fe layer grown on Vitrovac. Open circles correspond to an SP value in a single bunch measurements, i.e., each $120 \mathrm{~ns}$. The continuous line is the average on ten measurements.

of the Mott detector is shown in the top curves of Fig. 4, and the corresponding SP is shown in the lower panel. The 2.5\% variation in the intensities does not affect the SP, thus demonstrating that the SP is a genuine measure of the sample magnetization even in presence of an external field.

\section{A. Real time measurements: Magnetization reversal dynamics}

Independent SP data are collected and stored after each SR pulse therefore allowing, with a sampling frequency of $1 / 120 \mathrm{~ns}=8.33 \mathrm{MHz}$ to follow the reversal of the surface magnetization after the application of a reversal magnetizing field pulse. As an example of the reversal dynamics as a function of applied field strength we report in Fig. 5 the SP oscillations following the application of an oscillating magnetizing field with damped magnitude. The full line at the bottom represents the current through the magnetic circuit. The open symbols represent the single-pulse SP data and the continuous thick line is the result of an average on 10 repeated measurement cycles. The SP measurement obtained with a single SR pulse is sufficient to obtain the sample magnetization. As the magnitude of the applied magnetic field is reduced the corresponding SP reversal occurs with an increasing delay. The magnetization reversal process has been studied by the application of magnetic pulses with symmetric values (from $+H$ to $-H$ ) to a $2 \mathrm{~nm}$ Fe film on Vitrovac at $T=300 \mathrm{~K}$. In Fig. 6 we show the surface sensitive SP data (average of 300 repeated measurement cycles) compared with bulk sensitive measurements obtained by an induction search coil in identical experimental conditions. The data show that the total time required for completing the reversal is of the order of some $\mu$ s and strongly depends on the value of the applied field. The data also show that the magnetization reversal dynamics is different at the surface (Fe overlayer) with respect to the substrate (Vitrovac). The continuous lines through the data are obtained as best fit of a 


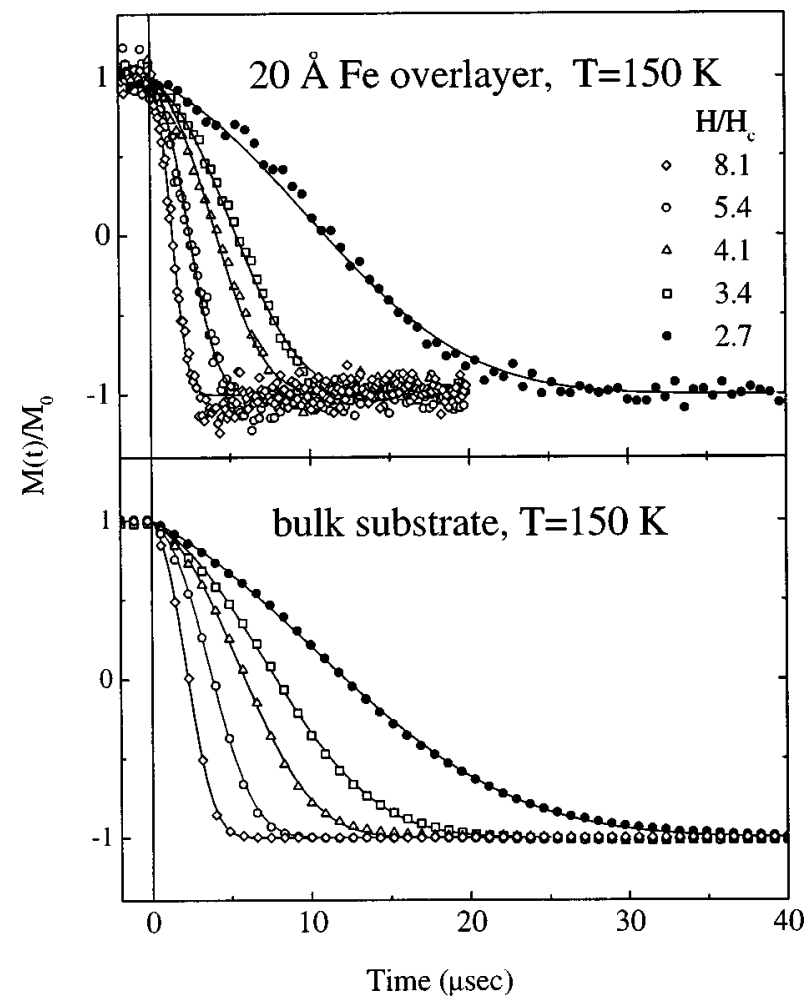

FIG. 6. Time-resolved SP measurements (average of 300 measurements) showing the magnetization reversal of a thin Fe layer $(20 \AA)$ deposited at RT on a Vitrovac ribbon. From left to right, the curves correspond to decreasing values (from 200 to $75 \mathrm{~mA}$ ) of the symmetric square $H$ field pulses. The magnetization value is reported as $M(t) / M_{0}$, where $M_{0}$ is the SP value in stationary conditions, i.e., $t=0$. At time below zero the film is magnetically saturated, i.e., in equilibrium condition. The continuous lines are the best fits obtained following the model of Ref. 15.

semiempirical model of the magnetization reversal for continuous films after Labrune et al. ${ }^{16}$ This model, likewise other similar models, ${ }^{17,18}$ describes the magnetization reversal with parameters representing the probability of nucleating a magnetic domain in the applied field direction, and the speed of growth of magnetic domains. Although such class of models with activation-barrier-like parameters brings only limited insight in the reversal process, the good fit obtained to our data allows to conclude that in this time domain of excitation and dynamics the magnetization reversal of an iron ATF is governed by domain processes. The dependence of the effective coercivity on the magnitude of the applied field is well observed in this time domain. ${ }^{19}$

A variety of experiments can be performed by controlling the following parameters: sign, magnitude, raise time, and duration of the applied magnetic field, sample thickness, coupling to the magnetic driver substrate, sample temperature, film continuity, etc. An example for asymmetric magnetic field pulses (different values for $+H_{1}$ and $-H_{2}$ ) of fixed magnitude, but variable time duration is shown in Fig. 7. In the figure the zero of the time scale indicates the field inversion from $+H_{1}$ to $-H_{2}$. The duration of the $+H_{1}$ field pulse is seen as negative time extent of the positive magnetization state. With applied $+H_{1}$ fields pulses of $0.12 \mu \mathrm{s}$ duration the surface magnetization remains basically unperturbed, but starting from $0.24 \mu \mathrm{s}$ of $+H_{1}$ duration the mag-

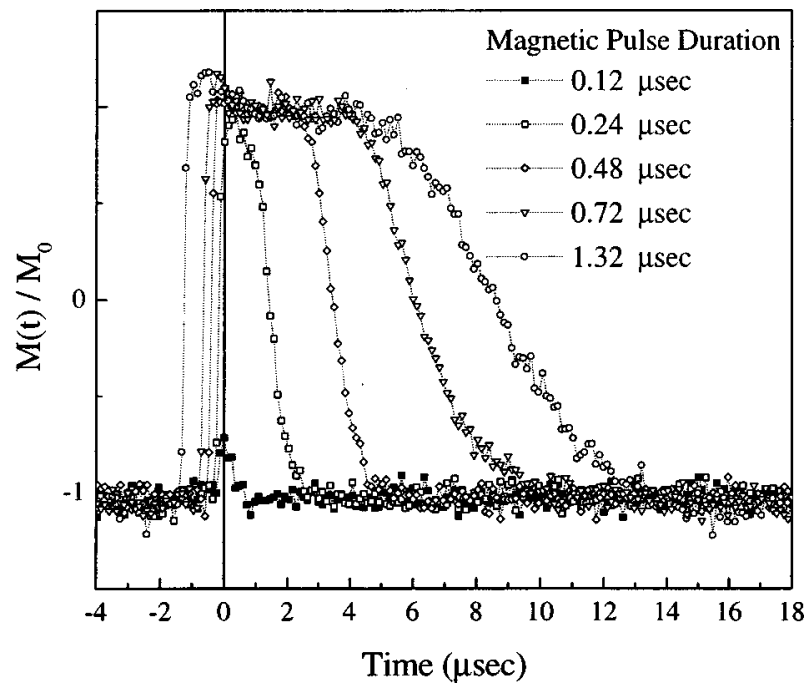

FIG. 7. Results for asymmetric $+H_{1}$ and $-H_{2}$ field pulses $\left(H_{1}=1.5 \mathrm{Oe}\right.$; $H_{2}=0.3 \mathrm{Oe}$ ) as a function of different pulse duration. No magnetization reversal is observed for a $0.12 \mu$ s time. A complete magnetization reversal is observed starting from a $0.24 \mu$ s duration of applied magnetizing field.

netization of the surface is completely reversed. It is interesting to observe that the dynamics of the reversal process strongly depends on the duration of the high field $+H_{1}$ pulse, i.e., of the previous history of the sample. These experiments show the importance of surface magnetic after effects. ${ }^{19,20}$

\section{B. Pump probe experiments: Fast magnetization reversal}

Introducing a delay between the SR clock and the magnetic field pulses, as explained above, pump-probe experiments can be performed and the time resolution can be pushed to its limit, i.e., the SR light pulsewidth $(\sim 500 \mathrm{ps}$ at SuperAco). The delay between pump ( $H$ field pulse) and probe (light pulse from the synchrotron) can be changed between each data acquisition producing reliable data sets with $1 \mathrm{~ns}$ resolution. The faster magnetization reversal that we could observe for a 2-nm-thick Fe film on Vitrovac was completed in a time $t \simeq 40 \mathrm{~ns}$ (see Fig. 8) which corresponds to $25 \mathrm{MHz}$ reversal rate. In this case the magnetization reversal time is shorter then the time needed to establish a steady current state within the applied field circuit, i.e., the magnetization change is driven by a changing applied field. In particular, for a magnetizing pulse of $H / H_{C}=140$ like that in Fig. 8, the complete reversal is obtained when the field has reached $60 \%$ of the final value. The pump-probe experiments with high time derivative of the applied magnetizing field $H$ must be understood by carefully considering the actual dynamics of the $H$ field pulse, i.e., the exact value of $d H / d t$ across the time interval of the magnetization reversal process. ${ }^{21}$

\section{CONCLUSIONS AND OUTLOOK}

We have here presented a new approach to the study of the dynamics of magnetization at surfaces based on the combination of SP measurements and time structure of SR. The 


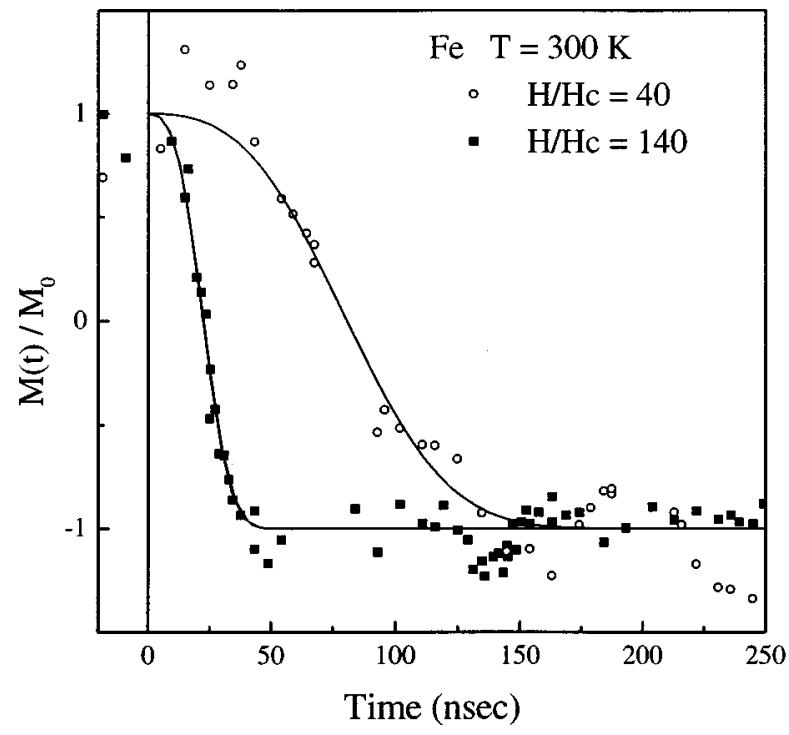

FIG. 8. $M(t) / M_{0}$ as a function of time, where $M_{0}$ is the SP value in stationary conditions, for a pump-probe experiment. Two kinds of magnetizing field pulses are shown: one with asymptotic values of $H / H_{C}=40$ (open circles) and with gradient of $51 \mathrm{MOe} \mathrm{s}^{-1}$. The second with an asymptotic value of $H / H_{C}=140$ (filled squares) and a gradient of $145 \mathrm{MOe} \mathrm{s}^{-1}$. The sample was a $2 \mathrm{~nm}$ Fe layer on Vitrovac at room temperature.

time resolution of the method is entirely set by the duration of the SR light pulse for pump-probe experiments and by the repetition rate of the SR pulses in real time experiments. In this last case the actual limiting rate of independent measurements is set by the dead time in between independently recordable signals which fact limits our current setup to a maximum real-time measurement rate of $12-15 \mathrm{MHz}$. The important aspect of our experimental setup is the intrinsic surface sensitivity of the SP measurement, which makes it the first synchrotron radiation-based time-resolved surface magnetometer. The whole domain of surface magnetic dynamics at the relevant time scales can be explored with the above-described experiments, including nonstationary states of a surface undergoing structural phase transitions. The few experimental results selected for this presentation all indicate that domain processes determine the surface magnetization reversal dynamics at $\sim 10 \mathrm{MHz}$ rate.

\section{ACKNOWLEDGMENTS}

The results presented here represent the achievement of the project, "Time Resolved Surface Magnetometry with Synchrotron Radiation," by G. Rossi and H. C. Siegmann, supported by the Swiss National Fund under Program 24. The authors gratefully acknowledge the continuous support of H. C. Siegmann and the collaboration of D. Guarisco and M. Liberati at different stages of the experimental work. G.P. acknowledges support by the EC Human Capital and Mobility Program.

${ }^{1}$ M. P. Sharrok, J. Appl. Phys. 76, 6413 (1994); P. J. Flanders and M. P. Sharrok, ibid. 62, 2981 (1987).

${ }^{2}$ R. H. Victora, Phys. Rev. B 63, 457 (1989).

${ }^{3}$ J. Pommier, P. Meyer, G. Penissard, J. Ferre P. Bruno, and D. Renaud, Phys. Rev. Lett. 65, 2054 (1990).

${ }^{4}$ P. J. Flanders and M. P. Sharrock, J. Appl. Phys. 62, 2918 (1987).

${ }^{5}$ B. Raquet, M. D. Ortega, M. Goiran, A. R. Fert, J. P. Redoules, R. Mamy, J. C. Ousset, A. Sdaq, and A. Khmou, J. Magn. Magn. Mater. 150, L5 (1995).

${ }^{6}$ F. B. Humphrey and E. M. Giorgy, J. Appl. Phys. 30, 935 (1959).

${ }^{7}$ W. D. Doyle, L. He, and P. J. Flanders, IEEE Trans. Magn. MAG-17, 3020 (1981).

${ }^{8}$ A. Vaterlaus, T. Beutler, and F. Meier, Phys. Rev. Lett. 67, 3314 (1991).

${ }^{9}$ D. Guarisco, Ph.D. thesis, ETH Zuerich, 1995.

${ }^{10}$ H. C. Siegmann, J. Phys.: Condens. Matter 4, 8935 (1992), and references therein.

${ }^{11}$ G. Rossi and F. Sirotti, in Magnetism and Synchrotron Radiation, ed. by E. Beaurepaire, B. Carriere, and J.-P. Kappler (Les Editions de Physique, Les Ulis, 1997), pp. 325-346.

${ }^{12}$ J. Kessler, Polarized Electrons, 2nd ed. (Springer, Berlin, 1995).

${ }^{13}$ H. C. Siegmann, F. Meier, M. Erbudak, and M. Landolt, Adv. Electron. Electron Phys. 62, 1 (1984).

${ }^{14}$ J. Kessler, Rev. Mod. Phys. 41, 3 (1969).

${ }^{15}$ G. Rossi, F. Sirotti, and G. Panaccione, in Core Level Spectroscopies for Magnetic Phenomena: Theory and Experiment, Vol. 345 of NATO-ASI Series B, edited by P. Bagus, G. Pacchioni, and F. Parmigiani (Plenum, New York, 1995), and references therein.

${ }^{16}$ M. Labrune, S. Andrieu, F. Rio, and P. Bernstein, J. Magn. Magn. Mater. 80, 211 (1989).

${ }^{17}$ P. Bruno, G. Bayreuhter, P. Beauvillain, C. Chappert, G. Lugert, D. Renard, J. P. Renard, and J. Seiden, J. Appl. Phys. 68, 5759 (1989).

${ }^{18}$ A. Lyberatos, J. Earl, and R. W. Chantrell, Phys. Rev. B 53, 5493 (1996).

${ }^{19}$ F. Sirotti and G. Rossi (unpublished).

${ }^{20}$ G. Bayreuter, P. Bruno, G. Lugaert, and C. Turtur, Phys. Rev. B 40, 7399 (1989).

${ }^{21}$ B. Raquet, R. Mamy, and J. C. Ousset, Phys. Rev. B 54, 4128 (1996). 